\title{
On the Achievable Rate in a D2D Cognitive Secondary Network Under Jamming Attacks
}

\author{
${ }^{\dagger b}$ S. Arunthavanathan, ${ }^{b}$ L. Goratti, ${ }^{b}$ L. Maggi, ${ }^{b}$ F. De Pellegrini, ${ }^{\dagger}$ S. Kandeepan \\ ${ }^{\dagger}$ School of Electrical Engineering, RMIT University, Melbourne, Australia, \\ ${ }^{b}$ CREATE-NET, Trento, Italy, \\ Email: s3391760@student.rmit.edu.au,kandeepan@ieee.org, \\ \{leonardo.goratti,lorenzo.maggi,francesco.depellegrini\}.create-net.org
}

\begin{abstract}
Ongoing developments of the LTE standard will allow for device-to-device (D2D) communications, which will enable direct connection of user equipments (UEs). Since UEs are becoming increasingly more powerful both in computational power and in the role they have in the network, a concrete threat is that a hand-held D2D-enabled device could be deployed to jam intentionally ongoing transmissions of other D2D users. In this context, a natural concern for operators will be the resilience of the legitimate user (LU) against a jammer's (J) attack. In this work, we model an LTE D2D system made of a pair of LUs and $\mathbf{a} \mathrm{J}$ that tries to impair their communication. We model the adversarial scenario between the transmitting $\mathbf{L U}$ and $J$ as a zero-sum game: in this game, J's target is to minimize the throughput of the legitimate D2D pair. We show the achievable channel rate of the D2D pair under jamming attacks and the existence of a Nash equilibrium. Finally, when both players learn each other strategy over time, e.g., employing fictitious play, such equilibrium becomes the system's operating point.
\end{abstract}

\section{INTRODUCTION}

The Third Generation Partnership Project (3GPP) has standardized the next generation of cellular technology, known as LTE and its advanced version (LTE-A), which is now approaching the mass market. The 3GPP community is currently defining the new features of LTE for communications systems beyond $4 \mathrm{G}$. One promising innovation that is expected to come with newer releases of LTE, namely Release 12 and 13, is provided by the $\mathrm{D} 2 \mathrm{D}$ communication mode. D2D appears promising in order to enable short range proximity services, offload traffic and perform efficient spectrum utilization.

However, this new paradigm will likely introduce strong requirements of coordination for legitimate D2D users. In particular handing over control of radio access to local UEs requires preventing destructive interference due to local transmissions that can potentially interfere with one another. Furthermore, with the increased programmability and computational power of UE terminals, a general threat in this scenario is represented by malicious users performing jamming attacks.

The aim in this paper is to provide a theoretical framework and novel performance evaluation tools able to quantify the impact of jamming for the emerging D2D paradigm. We consider the case of a legitimate transmitter/receiver pair subject to jamming attacks operated via the D2D communication mode. The transmitter is termed hereafter "legitimate", as it can access only the slots leased out locally by the primary communication system (i.e., cellular network).
The malicious user, namely the jammer, attempts to interfere with the physical resource blocks (PRBs) that the D2D transmitter exploits in the LTE radio frame. We hence consider the adversarial situation between $\mathbf{J}$ and the transmitting legitimate user, simply called LU. In the D2D scenario we assume that $\mathrm{J}$ and LU are both UEs and have similar type of cognitive capabilities, computational power, and radio characteristics. In addition, each of them may decide how to plan the transmission over each transmission time interval (TTI) in order to maximize their utility.

In the rest of the paper we provide a performance analysis based on the fundamental tradeoff that arises from combining physical layer considerations and link layer considerations. In particular, at each LTE radio frame, LU can select a subset of PRBs at random according to a frequency-time hopping scheme known by the receiver. The jammer, in turn, will try to hit a certain subset of PRBs with no prior knowledge on the hopping scheme. Actually, they both need to span the largest possible number of slots in order to escape/pursue the transmission of the opponent. But, under a finite power budget, they need at the same time to allocate a large enough power over each PRB to be effective, i.e., to transmit/interfere with enough energy per PRB.

The situation above represents a jamming game [1], [2] where the players' action is the number of PRBs randomly selected per frame. We formulate first the PHY layer analysis for the static case under fixed strategies for $\mathrm{J}$ and LU. Hence, the game between LU and $\mathrm{J}$ is described as a zero-sum game. Finally, we describe the system's dynamics under a learning scheme by which players react to each other strategy.

The remainder of this paper is organized as follows. Section II provides an in-depth explanation of the problem we aim to solve. Section III provides the system model and analysis. In Section IV we formulate the game theoretical approach considered in this paper. Section V illustrates and describes the simulation results obtained. Section VI shows the concluding remarks of the paper.

\section{PROBLEM FORMULATION}

In this work, we study a communication network in which a legitimate transmitter (or LU as mentioned before) is willing to communicate with the intended destination using D2D communication mode in the presence of a malicious device 
TABLE I

MAIN NOTATION USED THROUGHOUT THE PAPER

\begin{tabular}{|l|l|}
\hline Symbol & Meaning \\
\hline \hline LU & Transmitting legitimate user \\
\hline $\mathrm{J}$ & Jammer \\
\hline$M$ & Total number of available slots \\
\hline$m_{1}$ & Number of slots used by LU \\
\hline$m_{2}$ & Number of slots used by $\mathrm{J}$ \\
\hline$\pi^{(L U)}$ & $\begin{array}{l}\text { Transmission strategy LU. } \pi_{m_{1}}^{(L U)} \text { is the probability that LU } \\
\text { transmits on } m_{1} \text { slots }\end{array}$ \\
\hline$\pi^{(J)}$ & $\begin{array}{l}\text { Transmission strategy LU. } \pi_{m_{2}}^{(J)} \text { is the probability that } \mathrm{J} \\
\text { transmits on } m_{2} \text { slots }\end{array}$ \\
\hline$P_{\mathrm{LU}}$ & LU's transmitting power \\
\hline$P_{\mathrm{J}}$ & J's transmitting power \\
\hline$P_{\mathrm{d}}$ & $\begin{array}{l}\text { Probability that LU's signal is successfully detected by the } \\
\text { receiver }\end{array}$ \\
\hline$P_{i}$ & $\begin{array}{l}\text { Probability that there are } i \text { colliding slots, on which both LU } \\
\text { and J are both transmitting }\end{array}$ \\
\hline$A_{\mathrm{R}}$ & Expected achievable rate for LU \\
\hline
\end{tabular}

that attempts to impair the intended link. One possible example of a D2D communication protocol was presented, e.g., in [3], [4], [5]. The destructive effects of the jammer are measured at the location of the reference $\mathrm{D} 2 \mathrm{D}$ receiver. All devices are assumed hand-held battery operated terminals using LTE-A cellular technology with limited battery capabilities.

Since all the devices are battery operated they have to make judicious use of their power budget in order to maximize their utilities. From the perspective of the LU, this means maximizing the achievable transmission rate between source and destination, whereas for the jammer this implies destroying the legitimate communication link. We derived the probability that the intended destination can correctly receive the LU transmissions and we show how a zero-sum game can model the behavior of the network made of one legitimate D2D pair and the jammer. Actually, LTE-A allows scheduling resources inside the radio frame every TTI: we assume that a configuration of occupied PRBs remains constant during one LTE radio frame $(10 \mathrm{~ms})$. But, it can be changed in the next one (hopping scheme) so that the jammer cannot learn any specific pattern of the TTIs used by the LU transmitter. However, when opponents learn the number of employed PRBs of the adversary, e.g., by fictitious play, we can show that a Nash equilibrium is attained.

In the next section we develop the performance analysis of the system by encoding the impact of these constraints in our model. Thus, we can provide a framework to evaluate the best response of a legitimate user against jammers.

\section{SyStem Model AND ANALYSis}

The LTE radio technology relies on time-frequency slots to transmit information bits using orthogonal frequency division multiple access (OFDMA) for the downlink connection and single carrier frequency division multiple access (S-FDMA) for the uplink. The smallest unit of resource assignment in the LTE standard is the PRB, that is, the smallest bandwidth assignment to a user. Over time a PRB lasts half of a TTI (which corresponds to $1 \mathrm{~ms}$ ) but for the purpose of data exchange, a UE cannot use less than one entire TTI. One PRB contains 12 sub-carriers each spaced $15 \mathrm{kHz}$, which yields $180 \mathrm{kHz}$ of bandwidth [6]. Resources allocation is done by assigning to legitimate users time-frequency slots (or simply slots hereinafter) made of TTIs over time and PRBs in frequency. As shown in Figure 1, the LU receiver is located at the centre of the area of interest, which is assumed a disk of radius $R$, while the legitimate LU transmitter and the $\mathrm{J}$ are scattered at random inside the disk.

The total transmission powers available at the LU transmitter and jammer are denoted with $P_{\mathrm{LU}}$ and $P_{\mathrm{J}}$, respectively. At each LTE radio frame, the LU transmits over $m_{1}$ different TTIs and it utilizes an equally spread power $P_{\mathrm{LU}} / m_{1}$. Furthermore, we assume that the LU after selecting the number $m_{1}$ of slots it can change their position every LTE radio frame since the LTE system allows flexibly scheduling the resources. The jammer attempts to interfere with the transmission of the LU by transmitting at a power $P_{\mathrm{J}} / m_{2}$ equally distributed over $m_{2}$ slots.

We denote $M$ the total number of slots within a radio frame for a LTE system of bandwidth $W$. At each radio frame $\mathbf{J}$ and SU use $m_{1}$ and $m_{2}$ slots, respectively, where $1 \leq m_{1}, m_{2} \leq M$. Hence, they transmit over a set of $m_{1}$ and $m_{2}$ slots positioned at random over the LTE radio frame. We further assume that both J and SU are aware of each other's presence and they can estimate the number of channels used by the opponent at each radio frame.

As mentioned, beside interference both radio signals of the LU and $\mathrm{J}$ are assumed corrupted by a Rayleigh distributed fading. With interference, the probability the LU transmission can be correctly detected $\left(P_{\mathrm{d}}\right)$ at the receiver location can be written in terms of the signal-to-noise plus interference-ratio (SINR) as follows

$$
P_{\mathrm{d}}=\operatorname{Pr}(\mathrm{SINR} \geq \beta),
$$

where $\beta$ is the threshold for the detection.

Theorem 1: Under Rayleigh fading, the probability of successful detection $P_{\mathrm{d}}$ for a pair of legitimate users on a slot interfered by a jammer equals

$$
\begin{aligned}
P_{\mathrm{d}}=\exp \left(-\beta \frac{m_{1}}{\mathrm{SNR}}\right) \cdot & \frac{2}{\alpha}\left(\beta \frac{m_{1} P_{\mathrm{J}}}{m_{2} P_{\mathrm{LU}}}\right)^{\frac{2}{\alpha}}\left(\frac{r_{0}}{R}\right)^{2} \Gamma\left(1+\frac{2}{\alpha}\right) . \\
& \Gamma\left(\frac{-2}{\alpha}, \beta \frac{m_{1} P_{\mathrm{J}}}{m_{2} P_{\mathrm{LU}}}|h|^{2} \frac{r_{0}}{R}\right)^{\alpha},
\end{aligned}
$$

where $\alpha$ is the path-loss exponent, SNR the signal-to-noiseratio without interference and $r_{0}$ is the distance of the link between the LU transmitter and receiver.

The probability that the LU signal is successfully detected in the presence of Rayleigh fading only (i.e., without jamming) can be written as follows

$$
P_{d 0}=\operatorname{Pr}(\mathrm{SNR} \geq \beta) .
$$

The detailed proof of equation (1), which includes also equation (2) is postponed to the Appendix.

From the expression above, we notice that both LU and $\mathbf{J}$ allow finding a trade-off for the number $m_{1}$ and $m_{2}$ of slots they use. On the one hand, for the LU, opting for $m_{1}$ 


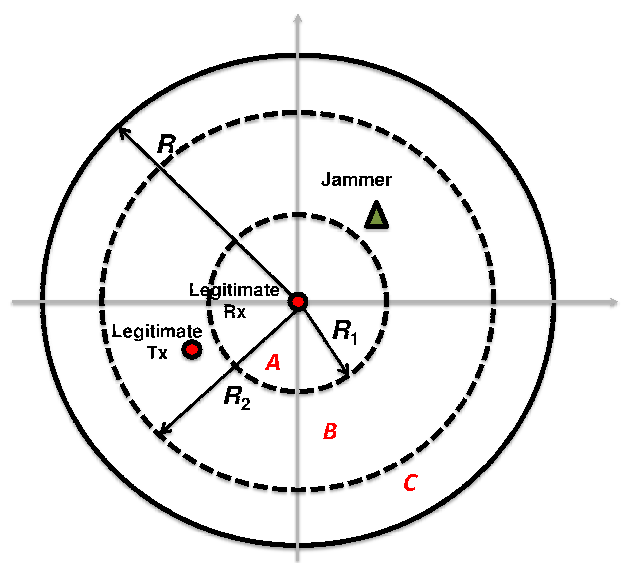

Fig. 1. System scenario: three logical areas define the operations of the system and the impact of the transmitter's and the jammer's strategies. Intuitively, a jammer in area A should hit a large fraction of slots irrespective of what the transmitter does. If $\mathrm{J}$ is in area $\mathrm{C}$, the transmitter should do the same. The intermediate case requires a game theoretical analysis.

close to $M$ results in a high probability of collision with the $\mathrm{J}$ and moreover in a low transmission power in each slot. On the other, choosing too small $m_{1}$ value allows using a higher transmission power per slot that reduces also the interference (unless $\mathrm{J}$ utilizes $m_{2}=M$ slots). However, choosing a too small $m_{1}$ value is often a conservative strategy. Conversely, from the perspective of the jammer, transmitting on a very small number $m_{2}$ of slots allows the $\mathrm{J}$ to interfere considerably the legitimate transmission over a small (on average) number of slots used by the LU. Increasing $m_{2}$, the jammer spreads the transmit power over a larger number of slots but at the penalty of a very low jamming effect.

The geometric interpretation of equation (1) is shown in Figure 1. The figure shows three different annulus inside the disk of area $\pi R^{2}$. Area $\mathbf{A}$ is the area where $P_{\mathrm{d}}$ is maximized; moving the LU transmitter away, Area $\mathbf{B}$ is the area where jamming interference starts effecting the reception of the legitimate D2D transmission since $P_{\mathrm{d}}$ is lower compared to the previous case and Area $\mathbf{C}$ where jamming attacks can disrupt the legitimate transmission since $P_{\mathrm{d}}$ lowers by moving the transmitter further away.

Irrespectively of the possible configurations described in Figure 1 , with a probability $P_{i}$, there exist $i$ slots where the jammer collides with the LU. Clearly, if $m_{1}>M-m_{2}$, there is collision on at least $m_{1}+m_{2}-M$ slots. Hence, we consider $i \geq \max \left(0, m_{1}+m_{2}-M\right)$. Moreover, $P_{i}=0$ $\forall i>\min \left(m_{1}, m_{2}\right)$. Therefore we can write the following

$$
P_{i}=\left(\begin{array}{c}
m_{1} \\
i
\end{array}\right)\left(\frac{m_{2}}{M}\right)^{i}\left(1-\frac{m_{2}}{M}\right)^{m_{1}-i},
$$

where $i=\max \left(0, m_{1}+m_{2}-M\right), \ldots, \min \left(m_{1}, m_{2}\right)$ and $P_{i}=$ 0 elsewhere.

We assume that LU aims at maximizing its utility that is the achievable rate $A_{\mathrm{R}}$, which is proportional to the expected number of PRBs successfully detected by the receiver. We denote such quantity as $A_{\mathrm{R}}\left(m_{1}, m_{2}\right)$, since it actually depends both on $m_{1}$ and on the number $m_{2}$ of slots chosen by $\mathrm{J}$.

We can now write the expression of the utility, that is the expected achievable rate $A_{\mathrm{R}}\left(m_{1}, m_{2}\right)$ for $\mathrm{LU}$, which is all the slots where the communication is successful:

$$
A_{\mathrm{R}}\left(m_{1}, m_{2}\right)=K \sum_{i=\max \left(0, m_{1}+m_{2}-M\right)}^{\min \left(m_{1}, m_{2}\right)} P_{i}\left[P_{\mathrm{d}} \cdot i+P_{\mathrm{d} 0} \cdot\left(m_{1}-i\right)\right] .
$$

where $K$ is a parameter which depends on the bandwidth of the LTE system contained in a frame and on the modulation scheme used. We will assign to $K$ a realistic value for LTE systems in the simulation results section. From, this point onwards, the dependency of $m_{1}$ and $m_{2}$ for $A_{\mathrm{R}}$ is dropped.

\section{ZERO-SUM GAME MODEL}

In this section we introduce a game-theoretical model for the situation where the action available for both LU and $\mathrm{J}$ is the number of PRBs used per frame. Our aim is to describe and predict the behaviour of two competing players, namely LU and J. The objective of LU is to achieve the highest possible transmission rate, which is proportional to its utility $U$. On the other hand, the malicious $\mathbf{J}$ intends to thwart LU's communication with the receiver. In this context, zero-sum games are a natural model [7], since $\mathrm{J}$ aims at minimizing $U$, i.e., maximizing $-U$, from which the games' name "zerosum".

\section{A. Static game: Nash Equilibrium}

We first study the conflict situation between LU and $\mathrm{J}$ over just one single transmission frame. This defines the single stage game setting. Players LU and $\mathrm{J}$ are thus have no information on the behaviour of their respective opponent, and they choose $m_{1}$ and $m_{2}$ independently of each other. Thus, the set of pure strategies for $\mathrm{J}$ and LU is the set $S=\{1, \ldots, M\}$.

Actually, we assume that both LU and J are both mixers, i.e., they can adopt mixed strategies for transmission. A mixed strategy is defined as a probability distribution according to which they select the number of their transmission slots at a given stage $m_{1}$ and $m_{2}$, respectively. We consider hence the standard uncorrelated case, where such distributions are independent of each other. Denote $\pi_{m_{1}}^{(L U)}\left(\pi_{m_{2}}^{(J)}\right.$, resp.) as the probability that LU $(\mathrm{J})$ transmits on $m_{1}\left(m_{2}\right)$ slots. We the denote by $A_{\mathrm{R}}\left(\pi^{(L U)}, \pi^{(J)}\right)$ as the expected value of $A_{\mathrm{R}}$ with respect to the (independent) transmission strategy of LU and J.i.e.,

$$
A_{\mathrm{R}}\left(\pi^{(L U)}, \pi^{(J)}\right)=\sum_{m_{1}=1}^{M} \sum_{m_{2}=1}^{M} \pi_{m_{1}}^{(L U)} \pi_{m_{2}}^{(J)} A_{\mathrm{R}}\left(m_{1}, m_{2}\right) .
$$

The adversarial situation that we have just described can be precisely modelled as a zero-sum game between LU and $\mathrm{J}$, which in this case are the two players of the game. A powerful concept in games is represented by the celebrated Nash equilibrium, that describes the situations in which none of the two agents can achieve a better utility by unilaterally changing its transmission strategy $\pi$ (i.e., when the other agent does not change its own strategy). 
Definition 1: The pair of transmission strategies $\left(\pi^{(L U) *}, \pi^{(J) *}\right)$ is said to be at Nash equilibrium whenever

$A_{\mathrm{R}}\left(\pi^{(L U)}, \pi^{(J) *}\right) \leq A_{\mathrm{R}}\left(\pi^{(L U) *}, \pi^{(J) *}\right) \leq A_{\mathrm{R}}\left(\pi^{(L U) *}, \pi^{(J)}\right)$

for all transmission strategies $\pi^{(L U)}, \pi^{(J)}$.

An early result in game theory is due to Von Neumann and relates to the existence of a unique value of the utility function that corresponds to the Nash equilibria of zero-sum games. In its general form, it holds the following

Theorem 2 ([8]): In zero-sum games, strategies at Nash equilibrium exist among mixed strategies. Furthermore, the value of the game $A_{\mathrm{R}}\left(\pi^{(L U) *}, \pi^{(J) *}\right)$ is unique.

We remark that not always it will be possible to find a Nash equilibrium among the so-called pure strategies. In fact, pure strategies are those where $m_{1}\left(m_{2}\right)$ which is selected with probability 1 by $L U(J)$. A NE in pure strategies is one for which each player uses pure strategies. In the other cases we define NE in mixed strategies. Later in the paper, we will show with numerical examples that both mixed and pure equilibria appear in the jamming game proposed in this paper.

\section{B. A learning strategy: fictitious play}

The Nash equilibria identified for the single stage case encompass the case when transmissions occur in one single frame.

Hereafter, we address the more realistic situation in which transmission occurs over multiple frames. We consider the case when both LU and $\mathrm{J}$ are capable to measure the respective opponent's behaviour over time. The results of the static case in the previous section are auxiliary for our development in the dynamic case.

At each frame $t=1,2, \ldots, \mathrm{LU}$ and $\mathrm{J}$ are able to change transmission strategy and select a different number of slots, $m_{1}(t)$ and $m_{2}(t)$ respectively, still according to some probability distribution.

We assume a situation of perfect mutual information, in which at frame $t$ both LU and $\mathrm{J}$ know exactly the number $m_{2}(k)$ and $m_{1}(k)$ utilized for transmission at frame $k=1, \ldots, t-1$ by the opponent $\mathrm{J}$ and LU, respectively. We now describe a learning process, in which the TU and $\mathbf{J}$ react optimally against the past behaviour of their respective opponent, by assuming that the opponent chooses the number of transmission slots at frame $t$ still according to the frequency of its own play up to frame $t-1$. This mechanism is called fictitious play [9].

Definition 2 (Fictitious play): Let $f_{m_{1}}^{(L U)}(t)\left(f_{m_{2}}^{(J)}(t)\right.$, resp.) the frequency up to time $t$ with which LU $(\mathrm{J})$ has transmitted on $m_{1}\left(m_{2}\right)$ slots, i.e.,

$$
\begin{aligned}
f_{m_{1}}^{(L U)}(t) & =\sum_{k=1}^{t} \mathbb{I}\left(m_{1}(k)=m_{1}\right) / t \\
f_{m_{2}}^{(J)}(t) & =\sum_{k=1}^{t} \mathbb{I}\left(m_{2}(k)=m_{2}\right) / t
\end{aligned}
$$

TABLE II

SYSTEM PARAMETERS ( [6], [5]).

\begin{tabular}{|c|l|l|}
\hline Parameter & Meaning & Value \\
\hline \hline$P_{\mathrm{LU}}$ & RF transmit power of UEs & $23 \mathrm{dBm}$ \\
\hline$W$ & Bandwidth & $1.4 \mathrm{MHz}$ \\
\hline$\beta$ & Threshold for detection & $1.5 \mathrm{~dB}$ \\
\hline$\alpha$ & Path-loss exp. & $2.01,4$ \\
\hline$N_{0}$ & Noise power spectr. density & $-174 \mathrm{dBm} / \mathrm{Hz}$ \\
\hline$\frac{r_{0}}{R}$ & Useful link distance & {$[0 ; 1]$} \\
\hline$\eta$ & LTE D2D protocol efficiency & 0.9 \\
\hline$b$ & bits/symbol (in 16-QAM) & 4 \\
\hline
\end{tabular}

where $\mathbb{I}(\cdot)$ defines the indicator function. By convention, we initialize $f_{m_{1}}^{(L U)}(0)=f_{m_{2}}^{(J)}(0)=1 / M$. From frame $t=1$ onwards,

$$
\begin{aligned}
& m_{1}(t)=\operatorname{argmax}_{m_{1}} A_{\mathrm{R}}\left(m_{1}, f_{m_{2}}^{(J)}(t-1)\right) \\
& m_{2}(t)=\operatorname{argmin}_{m_{2}} A_{\mathrm{R}}\left(f_{m_{1}}^{(L U)}(t-1), m_{2}\right)
\end{aligned}
$$

We remark that, in contrast with the static case described in the previous section, in fictitious play the strategies of the agents are no longer independent of each other, since each agent reacts to the past opponent's behaviour, in analogous ways.

It is well known [10] that the frequencies $f$ of the learning procedure of the fictitious play always converge to a (in general, mixed) equilibrium of the game.

Theorem 3 (Robinson 1951): If both LU and J adopt at each frame a fictitious-play type of transmission strategy, then their frequency of play converge to a Nash equilibrium strategies in the static case with probability 1 (w.p.1), i.e.,

$$
\begin{aligned}
\lim _{t \rightarrow \infty} f^{(L U)}(t) & =\pi^{(L U) *} \quad \text { w.p.1 } \\
\lim _{t \rightarrow \infty} f^{(J)}(t) & =\pi^{(J) *} \quad \text { w.p.1. }
\end{aligned}
$$

It is worth to remark that Thm. 3 states that, even though both players adopt at each stage a pure strategy drawn from set $S$, the frequencies by which those are played converge to the equilibrium of the game. As such, Thm. 3 is also a tool to determine the actual type of a NE, i.e., whether it is either mixed or pure.

\section{Simulation Results}

This section describes the results of our numerical simulations. The values of the parameters considered in the simulations are presented in Table II.

The parameter $\frac{r_{0}}{R}=0.5$ is taken into account, within area B of $P_{\mathrm{d}}$. A LTE device consists of 12 sub-carriers for the downlink, in which 7 symbols are carried by each carrier. Each LTE user is assigned $1 \mathrm{PRB}$ in bi-dimensional time and frequency of $10 \mathrm{~ms}$, comprising of 10TTIs of $1 \mathrm{~ms}$ each. This leads to the total number of resource elements(REs) in 


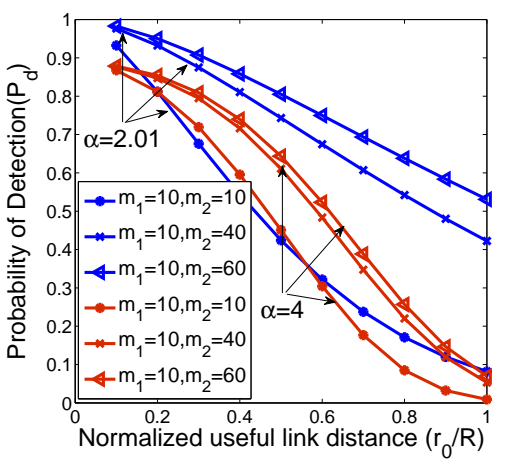

(a) $P_{d}$ vs. $\frac{r_{0}}{R}$ for fixed values of $\alpha$

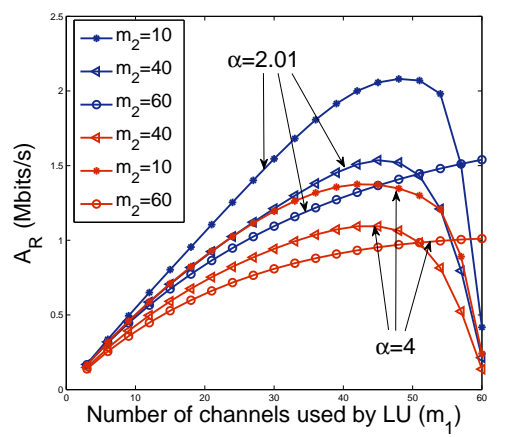

(b) $A_{\mathrm{R}}$ vs. $m_{1}$ for fixed $m_{2}$.

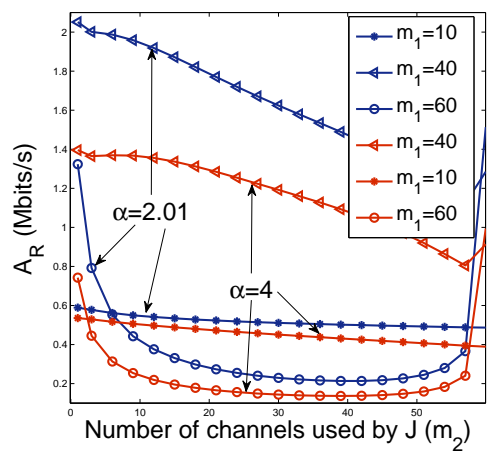

(c) $A_{\mathrm{R}}$ vs. $m_{2}$ for fixed $m_{1}$.

Fig. 2. Plots of the probability of detection $P_{\mathrm{d}}$ and of the achievable Rate $A_{\mathrm{R}}$ with parameters $\beta=1.5 d B, \frac{r_{0}}{R}=0.5$

a PRB: $12 \times 7=84$. The constant $K$ in the achievable rate formula in (4) is calculated as follows [5]:

$$
K=\eta \frac{84 \times 2 \times b}{10 m s}
$$

where $\eta$ is the D2D protocol efficiency and $b$ denotes the number of bits used in the 16-QAM, as listed in table II. $K$ is defined as the total number of data bits per second in 1 PRB.

Figure 2(a) shows that $P_{\mathrm{d}}$ reduces as $\frac{r_{0}}{R}$ increases, allowing the clarification of the three areas of LU transmission effectiveness as mentioned prior, for $\alpha=2.01$ and 4. One notes that $P_{\mathrm{d}}$ is smaller as $\alpha$ increases, due to the shifting from free space to indoors.

Simulations were also conducted to analyse the achievable rate of communication $A_{\mathrm{R}}$ between the pair of legitimate users. Fig. 2(b) (Fig. 2(c), resp.) shows $A_{\mathrm{R}}$ for fixed $m_{2}\left(m_{1}\right)$ and when $m_{1}\left(m_{2}\right)$ varies. We notice the trade-off that both LU and $\mathrm{J}$ have to face when choosing the number of slots to utilize, and which has been discussed in Section III. In Fig. 2(b) one may appreciate how the optimal $m_{1}$ for LU, that maximizes $A_{R}$, depends on the choice $m_{2}$ of J. Such optimal $m_{1}$ is called "best response" for LU against the action $m_{2}$ taken by J. Conversely, from Fig. 2(c) one observes the best response for $\mathbf{J}$, i.e., the optimal $m_{2}$ that minimizes the achievable rate against a certain $m_{1}$ selected by LU. If there exists a pair $\left(m_{1}, m_{2}\right)$ such that $m_{1}$ is best response against $m_{2}$ and, conversely, $m_{2}$ is best response against $m_{1}$, then $\left(m_{1}, m_{2}\right)$ is a pure Nash equilibrium.

Fig. 3, 4 show the frequency at which different number of slots have been chosen over time when both LU and $\mathrm{J}$ follow a fictitious play strategy. For Theorem 3, such frequencies converge to the Nash equilibrium of the static game, i.e., the game played over just one frame. In particular, from Fig. 3 we understand that for $M=60$ and a path loss $\alpha=2.01$, i.e. in outdoor environments, the Nash equilibrium is pure. Moreover, we observe that at Nash equilibrium $\mathrm{J}$ is forced to spread its available power on almost all (58 out of 60) the RBs available. However, it is erroneous to claim that $\mathrm{J}$ is always better off by jamming almost all slots whatever the strategy for LU is, as it is clear from Fig. 2(c).

As Theorem 2 claims, in general we should expect the emer- gence of mixed Nash equilibria, of whom pure ones are just a special case. In mixed equilibria, $\mathrm{LU}$ and $\mathrm{J}$ choose the number of RBs according to some independent probability distribution. Fig. 4 shows that for $M=60$ and a path loss exponent $\alpha=4$, i.e. in indoor environments, LU picks $m_{1}=40$ and $m_{1}=41$ with probability around 0.3 and 0.7 , respectively. In this case, $\mathrm{J}$ is still forced to jam on almost all the available RBs, by selecting $m_{2}=58$ and $m_{2}=59$ with probability around 0.8 and 0.2 , respectively.

Finally, we also observed that if the parameter $\frac{r_{0}}{R}$ tends to 1, i.e. Area $\mathrm{C}$ is approached, then $P_{\mathrm{d}}$ decreases, thus leading to the emergence of mixed NE strategies.

\section{CONCLUSiOnS}

In this paper we tackled the challenging problem that might arise when newer releases of LTE technology will allow users to communicate via a D2D communication mode. In this context, some devices might develop a malicious behaviour by starting intentionally jamming other legitimate pair of user equipments.

Assuming that a pair of legitimate users is aware of the presence of the jammer, we analyse the adversarial situation between the transmitting legitimate user (LU) and the jammer (J) by utilizing a game-theoretical framework, and more specifically via zero-sum games. At each LTE frame, J and LU are able to choose the number of time-frequency slots to transmit on, out of the $M$ available ones. J's objective is to minimize the expected achievable rate for the pair of LUs. In this context, we compute the Nash equilibrium of the game, describing the pair of strategies for $\mathrm{LU}$ and $\mathrm{J}$ from which it is not beneficial for either of the conflicting agents to unilaterally deviate. The Nash equilibrium can be considered as the operating point of the system, and it can be reached asymptotically via a simple learning strategy by both users, called fictitious play. As a matter of fact, it also prescribes the max-min strategy for LU, being the best conservative strategy for LU against the most harmful behaviour of $\mathrm{J}$.

Numerical simulations suggest that, for ranges of parameters of practical interest, the best strategy for $\mathrm{J}$ is to jam nearly as many slots as possible, while LU should utilize a significant fraction of the available slots. 

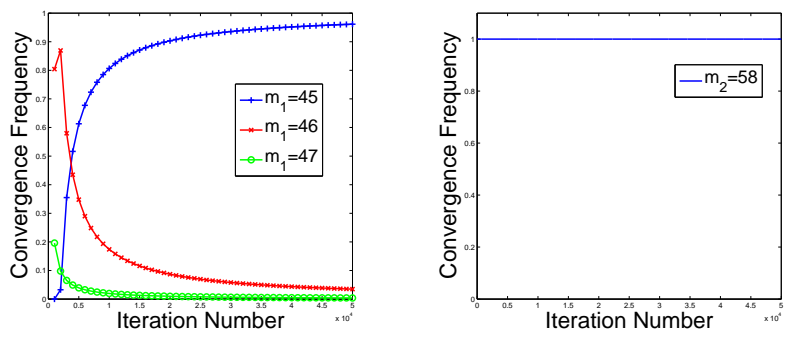

Fig. 3. Frequency of play when both LU and $\mathrm{J}$ choose the number of slots $m_{1}$ and $m_{2}$ to transmit on according to a fictitious play strategy, with $\alpha=2.01$, $\beta=1.5 d B, \frac{r_{0}}{R}=0.5$.
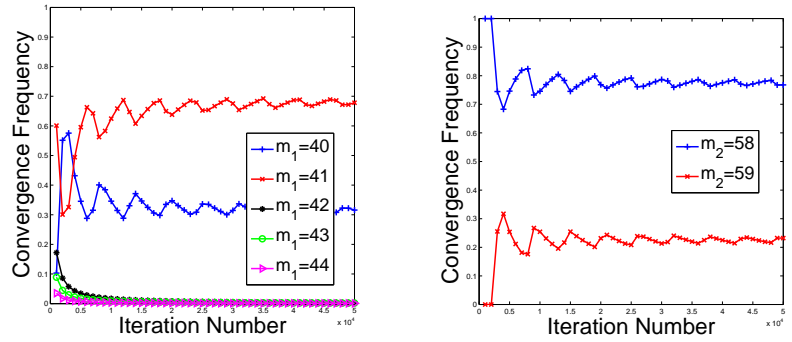

Fig. 4. Frequency of play when both LU and $\mathrm{J}$ choose the number of slots $m_{1}$ and $m_{2}$ to transmit on according to a fictitious play strategy, with $\alpha=4$, $\beta=1.5 d B, \frac{r_{0}}{R}=0.5$.

As a by-product, we also computed the probability that the receiving LU can successfully decode the useful LU's signal in the presence of a Rayleigh distributed fading channel with J's interference.

\section{ACKNOWLEDGMENT}

The research of S. Arunthavanathan, L. Goratti, and S. Kandeepan, leading to these results, has received partial funding from the EC 7 Framework Programme (FP7-2011-8) under the Grant Agreement FP7-ICT-318632. The work of F. De Pellegrini and L. Maggi has been partially supported by the European Commission within the framework of the CONGAS project FP7-ICT-2011-8-317672, see www.congas-project.eu.

\section{APPENDIX}

\section{PROOF OF THEOREM 1}

Proof: Let us assume that one channel occupied by LU is interfered by $\mathrm{J}$. The power gain coefficient of the channel in question is simply denoted as $|h|^{2}$. Then,

$$
\begin{aligned}
& S I R=\frac{\frac{1}{m_{1}} P_{\mathrm{T}}\left|h_{0}\right|^{2}}{\frac{1}{m_{2}} P_{\mathrm{J}}|h|^{2}} \frac{r_{0}^{-\alpha}}{r^{-\alpha}} \\
& \bar{P}_{\mathrm{d}}^{*}=\operatorname{Pr}\left(\left|h_{0}\right|^{2} \geq \beta \frac{m_{1} P_{\mathrm{J}} \mid h_{\mid}^{2} r^{-\alpha}}{m_{2} P_{\mathrm{T}} r_{0}^{-\alpha}}\right)
\end{aligned}
$$

The following expression for $\bar{P}_{\mathrm{d}}^{*}$ considers a 2-dimensional area of transmission.

$$
\bar{P}_{\mathrm{d}}^{*}=\int_{R_{1}}^{R_{2}} e^{-\beta \frac{m_{1} P_{\mathrm{J}}|h|^{2} r^{-\alpha}}{m_{2} P_{\mathrm{T}} r_{0}^{-\alpha}}} \frac{d r^{d-1}}{R^{d}} d r
$$

Performing integration by substitution, we use the change of variable $t=\beta \frac{m_{1} P_{\mathrm{J}}}{m_{2} P_{\mathrm{T}}}|h|^{2} r^{-\alpha} r_{0}{ }^{\alpha}$. Hence, the integral can be rewritten as

$$
\begin{aligned}
\bar{P}_{\mathrm{d}}^{*}= & \frac{d}{\alpha}\left(\frac{r_{0}}{R}\right)^{d}\left(\beta \frac{m_{1} P_{\mathrm{J}}}{m_{2} P_{\mathrm{T}}}\right)^{\frac{d}{\alpha}}|h|^{\frac{2 d}{\alpha}} . \\
& {\left[\int_{t_{2}}^{\infty} e^{-t} t^{\frac{-d}{\alpha}-1} d t-\int_{t_{1}}^{\infty} e^{-t} t^{\frac{-d}{\alpha}-1} d t\right], }
\end{aligned}
$$

where the lower limit $t_{1}=\beta \frac{m_{1} P_{\mathrm{J}}}{m_{2} P_{\mathrm{T}}}|h|^{2} m_{1}^{-\alpha} r_{0}^{\alpha}$ and the upper limit $t_{2}=\beta \frac{m_{1} P_{\mathrm{J}}}{m_{2} P_{\mathrm{T}}}|h|^{2} R_{2}^{-\alpha} r_{0}^{\alpha}$.

We are now in the position to compute $\bar{P}_{\mathrm{d}}^{*}=\mathbb{E}_{h}\left(P_{\mathrm{d}}\right)$ by removing the conditioning on the channel fading and assuming $R_{1}=0$ and $R_{1}=R$ as follows

$$
\begin{aligned}
& \bar{P}_{\mathrm{d}}^{*}=\frac{d}{\alpha}\left(\beta \frac{m_{1} P_{\mathrm{J}}}{m_{2} P_{\mathrm{T}}}\right)^{\frac{d}{\alpha}}\left(\frac{r_{0}}{R}\right)^{d} \Gamma\left(1+\frac{d}{\alpha}\right) . \\
& {\left[\Gamma\left(\frac{-d}{\alpha}, \beta \frac{m_{1} P_{\mathrm{J}}}{m_{2} P_{\mathrm{T}}}|h|^{2} \frac{r_{0}}{R}\right)^{\alpha}\right], }
\end{aligned}
$$

where in the Rayleigh distribution we assume $\sigma=\frac{1}{\sqrt{2}}$.

The detection probability shown in equation (1) is obtained by noticing that in the case of Rayleigh fading equation (2) is simply $P_{\mathrm{d} 0}=\exp \left(-\beta \frac{N_{0} W}{P_{\mathrm{t}} / m_{1} r_{0}^{-\alpha}}\right)$. The final expression can be then obtained by multiplying $P_{\mathrm{d}}=P_{\mathrm{d} 0} \cdot \bar{P}_{\mathrm{d}}^{*}$

\section{REFERENCES}

[1] B. Wang, Y. Wu, and K. J. R. Liu, "Optimal Power Allocation Strategy against Jamming Attacks using the Colonel Blotto Game," IEEE GLOBECOM Proc., pp. 1-5, 2009.

[2] K. R. L. Y. Wu, B. Wang and T. C. Clancy, "Anti-jamming Games in Multi-channel Cognitive Radio Networks," IEEE Commun., vol. 30, no. 1, pp. 1-12, 2012.

[3] B. Zhou, H. Hu, S.-Q. Huang, and H.-H. Chen, "Intracluster Deviceto-Device Relay Algorithm with Optimal Resource Utilization," IEEE Trans. on Veh. Technol., vol. 62, no. 5, pp. 2315-2326, 2013.

[4] B. Raghothaman, E. Deng, R. Pragada, G. Sternberg, T. Deng, and K. Vanganuru, "Architecture and Protocols for LTE-based Device to Device Communication," in Computing, Networking and Communications (ICNC), 2013 Intl. Conf. on, 2013, pp. 895-899.

[5] L. Goratti, K. M. Gomez, R. Fedrizzi, and T. Rasheed, "A Novel Deviceto-Device Communication Protocol for Public Safety Applications," IEEE, 2013

[6] S. Sesia, I. Toufik, and M. Baker, The UMTS Long Term Evolution From Theory to Practice, Feb. 2009, Wiley, Second Edition.

[7] E. Altman, K. Avrachenkov, and A. Garnaev, "Fair resource allocation in wireless networks in the presence of a jammer," Performance Evaluation, vol. 67, no. 4, pp. 338-349, 2010.

[8] R. Myerson, Game Theory: Analysis of Conflict, 1997.

[9] G. Brown, Iterative Solutions of Games by Fictitious Play In Activity Analysis of Production and Allocation, 1951.

[10] J. Robinson, "An iterative method of solving a game," Annals of Mathematical Statistics, vol. 54, pp. 296-301, 1951. 